\title{
Simulation of Impinging Cooling Performance with Pin Fins and Mist Cooling Adopted in a Simplified Gas Turbine Transition Piece
}

\author{
Tao Xu, Hang Xiu, Junlou Li, Haichao Ge, Qing Shao, Guang Yang, and Zhenglei Yu \\ College of Mechanical Science \& Engineering, Jilin University, Changchun 130025, China
}

Correspondence should be addressed to Zhenglei Yu; yuzhenglei@hotmail.com

Received 6 December 2013; Accepted 27 January 2014; Published 9 March 2014

Academic Editor: Raseelo Joel Moitsheki

Copyright (C) 2014 Tao Xu et al. This is an open access article distributed under the Creative Commons Attribution License, which permits unrestricted use, distribution, and reproduction in any medium, provided the original work is properly cited.

\begin{abstract}
The gas turbine transition piece was simplified to a one-four cylinder double chamber model with a single row of impinging holes in the outer wall. Heat transfer augmentation in the coolant chamber was achieved through the use of pin fin structure and mist cooling, which could increase the turbulence and heat transfer efficiency. The present research is focused on heat transfer and pressure characteristics of the impinging cooling in the coolant chamber using FLUENT software. With the given diameter of impinging hole, pin fin diameter ratios $D / d$ have been numerically studied in ranges from 1 to 2 . Three different detached $L$ were simulated. The impinging cooling performance in all cases was compared between single-phase and two-phase (imported appropriate mist) flow in the coolant chamber. All the simulation results reveal that the factors of $L$ and $D / d$ have significant effects on the convective heat transfer. After the pin fin structure was taken, the resulting temperature decrease of $38.77 \mathrm{~K}$ at most compared with the result of structure without pin fins. And with the mist injecting into the cooling chamber, the area weighted average temperature got a lower value without excess pressure loss, which could satisfy the more stringent requirements in engineering.
\end{abstract}

\section{Introduction}

Low installation cost and higher output have made gas turbine a popular power plant in the modern industries. Gas turbines have been widely used in the domain of aerospace and ship propulsion for more than half a century. To meet the increasing requirement of power, research and improvement of heat efficiency of gas turbines have become the hottest research focus. To improve the thermal efficiency and output of gas turbines, the temperature of working fluid is usually elevated higher than the metal melting. Spontaneously, the components of gas turbine, such as combustion chamber, combustor transition pieces, and turbine blades, need to be protected from the high temperature gas by cooling technology. A wide variety of cooling technologies including film cooling and impinging cooling have been successfully used in cooling of these hot components for a few decades.

Impinging jets are used in many applications for providing high heat/mass transfer rates. Compared to other heat or mass transfer arrangements that do not employ phase change, the jet impingement device offers efficient use of the fluid and high transfer rates. In turbine applications, jet impingement may be used to cool several different sections of the engine such as the combustor case, turbine case/liner, and the critical high temperature turbine blades [1]; also the transition piece used this cooling method mostly. General applications and performance of impinging jets had been discussed in a number of reviews [2-4]. The jet impingement angle has an effect on heat transfer and was studied frequently $[2,3]$. Besides, some other parameters also have important effects on the impinging characteristics. Dano et al. [5] researched the effects of nozzle geometry on the flow characteristics and heat transfer performance. Cheong et al. [6] experimentally measured local heat transfer coefficients under an impinging jet with low nozzle-to-plate, $z / d$, and spacings.

Meanwhile, pin fin cooling is often employed to protect the hot sections from thermal degradation while extending the durability. It is a commonly used method in the trailing edge of the gas turbine blades and many channel flows [7-10]. Horbach et al. [8] describe an experimental study on trailing edge film cooling using coolant ejection. The experimental test investigated the effects of different pin fins geometric 
configurations, and the result shows that the elliptic pin fins have a strong effect on discharge behavior as well as on cooling effectiveness and heat transfer.

In the literature $[9,10]$, experimental and numerical studies of heat transfer performance in channels with pin fins were conducted by air. The results show that the channels with pin fins had a heat transfer coefficient that was twice that of the channels without pin fins. The numerical computations showed the same trends as experimentally observed by the heat transfer enhancement through pin fin structure adopted. Heat transfer from pin fin parameters is a subject of high importance with many engineering applications.

Most of the above works are based on geometric parameters of the cooling structure; in real applications, new cooling techniques are another way to get enhancement of the conventional impinging cooling. Wang and Li [11] proposed a promising technology to enhance air film cooling with mist (small water droplets) injection. Each droplet plays the part of cooling sink, and it flies a distance before it completely vaporizes. And the droplet evaporation plays an important role in reducing the temperature near the hot wall.

Li and Wang [12] conducted their first numerical simulations of air/mist film cooling. They showed that injecting some appropriate mist in the air could enhance the cooling effectiveness to about 30-50\%. After that they [13] continued a more fundamental study on investigating the effect of various models on the computational results including the turbulence models, dispersed phase modeling, and different forces models (Saffman, thermophoresis, and Brownian).

Whereas all the studies mentioned above about mist cooling focus on film cooling style and the previous literatures about impinging cooling and pin fin cooling structure mainly focus on gas turbine blades structure, electronic equipment, and many other channel flow. Although Yu et al. [14] introduce the mist into impinging cooling technology, the cooling structure does not contain pin fin structure. And those literatures about pin fins focus on some pin fin structures, such as cross-sectional shape, detached space between the pin tip and the end wall, which is a limited work about the impinging characteristics through combining pin fin structures, mist cooling, and impingement.

Based on the aforementioned cooling structure and mist film cooling mechanisms, limited work is given to investigate the impinging cooling performance in this study. A model of a one-fourth cylinder is designed which could simplify the impinging structure and performance used in gas turbine transition piece. The objective of this paper is to use CFD simulation to investigate impinging cooling performance with pin fin importing to the coolant chamber. Two significant effect factors in combination with impinging cooling and pin fin structure were investigated using numerical simulations. All the cases were simulated in two situations; one is only air in the coolant and another is air with mist together in the cooling chamber, which is helpful in getting a better cooling effectiveness.

Accordingly, the main objectives of the investigation are as follows. (1) $D / d$ analysis: with the given diameter of impinging hole, $d=10.26 \mathrm{~mm}$, pin fin diameter ratio $\mathrm{D} / \mathrm{d}$ has been numerically studied in three different values, $1,1.5$, and 2. (2) Detached space analysis: three different detached spaces from pin fin array to impinging hole array (mark as $L=34,51$, and $68 \mathrm{~mm}$ ) were simulated. (3) Comparison of results: the temperature of the inner wall, cooling effectiveness, and contours of velocity in different cases. (4) Discuss the impinging cooling performance between single-phase and two-phase flow in the cooling chamber with import appropriate mist (small droplets) into the coolant (air).

\section{Numerical Model}

2.1. Geometric Configuration. The transition piece was simplified to a one-fourth cylinder, which could simulate the transition piece's structure and performance $[15,16]$. The discrete coolant jets, forming a protective film chamber on the side of transition piece, are drawn from the upstream compressor in an operational gas turbine engine. The coolant flows fed through internal passages with surface holes. From the supply plenum, the coolants ejected through three discrete impinging holes over the external boundary layer against the local high thermal conduction on the other side of the transition piece. In the downstream of these impinging holes, three pin fins were brought into the cooling chamber. A schematic diagram of the flow domain along with boundary conditions and dimensions is given in Figure 1.

As shown in the figure, the one-fourth cylinder model has two layers of chambers with a length of $1050 \mathrm{~mm}$, and the outer chamber is right the domain full of coolant, which is our major research object. The dimensions of the chambers are, respectively, defined as an outer radius and an inner radius of $200 \mathrm{~mm}$ and $162 \mathrm{~mm}$. In the diagram, one side of the coolant chamber is closed; contrarily, both sides of the mainstream chamber as the inner chamber are opened in which gas could flow through from one side to the other. The three holes distributed uniformly in one row along the circumferential direction in the surface of the outer wall. The distance between holes and the pin fins is marked as a variable parameter $L$. The diameter of all the impinging holes is about $10.26 \mathrm{~mm}$, that is, $d=10.26 \mathrm{~mm}$. Three groups of pin fin diameter ratio $D / d$ (where $D$ means pin fin diameter) have been constituted, which is set to be $1,1.5$, and 2 .

\subsection{Numerical Method}

2.2.1. Governing Equations. The present impinging and pin fin cooling study involves a flow which is steadied, Newtonian, three-dimensional, incompressible, and turbulent. For solving this state of fluid, mass, energy, and species, transport equations need to be solved. The continuity, momentum, and energy equations are given by [16].

$$
\begin{gathered}
\frac{\partial}{\partial x_{i}}\left(\rho u_{i}\right)=0, \\
\frac{\partial}{\partial x_{i}}\left(\rho u_{i} u_{j}\right)=\rho \vec{g}_{i}-\frac{\partial P}{\partial x_{i}}+\frac{\partial \tau_{i j}}{\partial x_{j}}+F_{i}, \\
\rho\left(u_{i} \frac{\partial e}{\partial x_{i}}\right)=-\frac{\partial}{\partial x_{k}}\left(-k \frac{\partial T}{\partial x_{k}}\right)-P \frac{\partial u_{k}}{\partial x_{k}}+\varphi,
\end{gathered}
$$




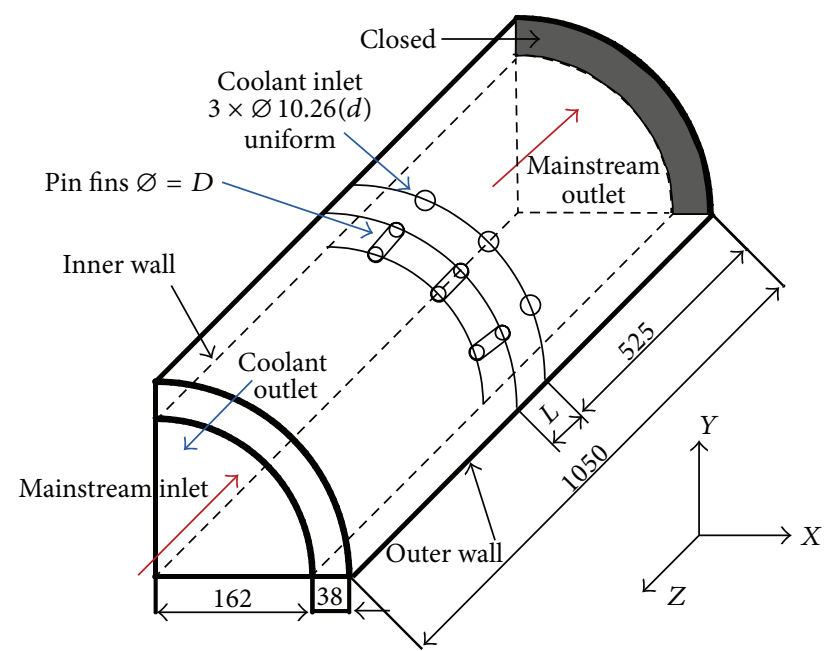

(a)

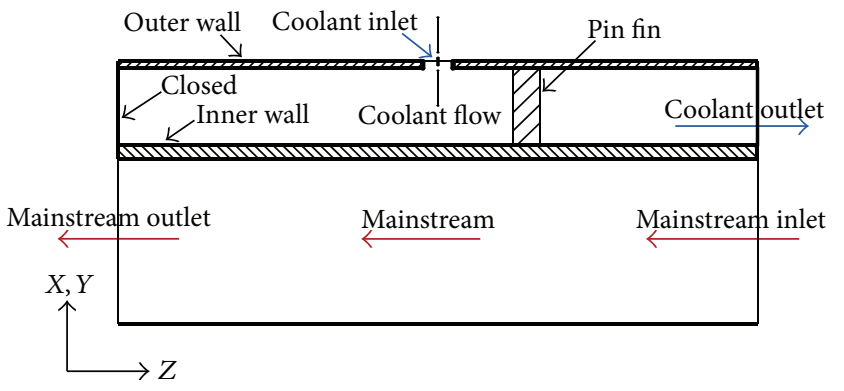

(b)

FIGURE 1: Computational domain showing boundary conditions.

where $P$ is the total pressure, $\tau_{i j}$ is the stress tensor, $\rho \vec{g}_{i}$ and $F_{i}$ are the gravitational body force and external body forces, $\varphi$ is the viscous heating dissipation, and the heat flux is given by Fourier's law.

2.2.2. Turbulence Model. A wide used turbulence model is the realizable $k-\varepsilon$ turbulence model which is a relatively recent development model. The term "realizable" means that the model satisfies certain mathematical constraints on the Reynolds stresses, consistent with the physics of turbulent flows [17]. The benefit of selecting this model is that it more accurately predicts the spreading rate of jets and it also provides superior performance for rotation, separation, and recirculation flows. In this model, the $k$ equation is the same as in RNG model; however $C_{\mu}$ is not a constant and varies as a function of mean velocity field and turbulence (0.09 in loglayer of $S(k / \varepsilon)=3.3$ and 0.05 in shear layer of $S(k / \varepsilon)=6$ ). The equation is based on a transport equation for the meansquare vorticity fluctuation [18] as follows:

$$
\rho \frac{D \varepsilon}{D t}=\frac{\partial}{\partial x_{j}}\left[\left(\mu+\frac{\mu_{t}}{\sigma}\right) \frac{\partial \varepsilon}{\partial x_{j}}\right]+C_{1} S \rho \varepsilon-C_{2} \frac{\rho \varepsilon^{2}}{k+\sqrt{v e}},
$$

where $C_{1}=\max [0.43, \eta /(\eta+1)]$ and $C_{2}=1.0$. This model is used with standard wall functions to predict flow structure and heat transfer over the inner wall cooling surface.

2.2.3. Dispersed-Phase Model/Water Droplets (Mist). Based on the Newton's second law, the droplet trajectory is traced by

$$
m_{p} \frac{d v_{p}}{d t}=\Sigma F,
$$

where $m_{p}$ is the droplet mass and $v_{p}$ is the droplet velocity. $\Sigma F$ is the combined force on the droplet particle, which normally includes the hydrodynamic drag, gravity, and other forces.
The energy balance for any individual droplet can be given as the following equation:

$$
m_{p} c_{p} \frac{d T}{d t}=\pi d^{2} h\left(T_{\infty}-T\right)+\frac{d m_{p}}{d t} h_{f g},
$$

where $h_{f g}$ is the latent heat. The convective heat transfer coefficient $(h)$ can be obtained with an empirical correlation [19].

The mass change rate/vaporization rate in (4) is governed by concentration difference between droplet surface and air stream as follows:

$$
-\frac{d m_{p}}{d t}=\pi d^{2} k_{C}\left(C_{S}-C_{\infty}\right),
$$

where $k_{C}$ is the mass transfer coefficient and $C_{S}$ is the vapor concentration at the droplet surface, which is evaluated by assuming that the flow over the surface is saturated. $C_{\infty}$ is the vapor concentration of the bulk flow. When the droplet temperature reaches the boiling point, the following equation can be used to evaluate its evaporation rate [20]:

$$
\begin{aligned}
-\frac{d m_{p}}{d t}= & \pi d^{2}\left(\frac{\lambda}{d}\right)\left(2.0+0.46 \mathrm{Re}_{d}^{0.5}\right) \\
& \times \ln \frac{\left(1+c_{p}\left(T_{\infty}-T\right) / h_{f g}\right)}{c_{p}},
\end{aligned}
$$

where $\lambda$ is the gas/air heat conductivity and $c_{p}$ is its specific heat. The instantaneous turbulence effect on the dispersion of particles can be considered by using stochastic tracking. The droplet trajectories are calculated with the instantaneous flow velocity $\left(\bar{u}+u^{\prime}\right)$, and the velocity fluctuations are then given as follows:

$$
u^{\prime}=\varsigma\left(\overline{u^{\prime 2}}\right)^{0.5}=\varsigma\left(\frac{2 k}{3}\right)^{0.5}
$$


where $\varsigma$ is a normally distributed random number. This velocity will apply during the characteristic lifetime of the eddy te, a time scale calculated from the turbulence kinetic energy and dissipation rate. After this time period, the instantaneous velocity will be updated with a new $\varsigma$ value until a full trajectory is obtained. A more detailed discussion about the stochastic method and the two stages of evaporation is given by Li and Wang [21], and more numerical details are given in FLUENT [17].

2.3. Boundary Condition Setup. In the case of impinging cooling simulation, boundary conditions are applied to specify the flow and thermal variables. Figure 1(a) discloses the boundary conditions used in the model in which the coolant and gas are moving along, respectively, in the two layers of chambers in opposite directions. In the cooling chamber, the coolant is considered to be two situations. One is single-phase analysis. The coolant is only air, while velocity and temperature are set on the jet holes, with pressure on the exit. Another situation is two-phase flow in the cooling chamber, which is considered mist added in the air. Additional boundary conditions for mist injections are specified. The size of droplets is uniformly given as 5 microns, and the masses of water droplets are $0.003 \mathrm{~kg} / \mathrm{s}$. The similar conditions have been successfully used in film cooling technology by Subbuswamy and Li [22]. The solid walls are assigned as "reflect" boundary condition, which enables the droplets to elastically rebound once hitting the wall. The outer inlet is specified as "escape" boundary condition for droplets so that they can enter into the cooling chamber from the inlet surface.

In the chamber of mainstream, the gas flow is a mixture of $\mathrm{O}_{2}, \mathrm{H}_{2} \mathrm{O}, \mathrm{CO}_{2}$, and $\mathrm{N}_{2}$, as well as some rare gasses, which is confirmed based on several real applications. Uniform mass flux rate of $31.46 \mathrm{~kg} / \mathrm{s}$ is assigned to the inlet of the gas chamber, and other details about boundary conditions are listed in Table 1. Assumption of the solid wall of the model is formed with a material of Nimonic 263, which could get the information from the Internet [23].

\section{Meshing and Simulation Procedures}

To conduct numerical simulations, structured grids are used in this study. As shown in Figure 2, the structured meshes are generated to two domains, which is on behalf of the coolant air chamber and gas chamber separately. In the 3D cases, the grid sensitivity research started from 150,000 meshes until the temperature result changed less than $1 \%$ when the total number of the cells for the 3D domains is about 500,000. All the cases concerning different parameters and conditions are meshed with the same proper setup on the boundary and got the similar total mesh number. Local grid refinement is used near the holes and pin fin regions. For all cases, all nodes on the inner wall surface have the $y$ plus value smaller than 300 .

This study is using a commercial CFD code based on the control-volume method, ANSYS-FLUENT 12.1, which is used in order to predict temperature, impingement cooling effectiveness, and velocity fields. All runs were made on a PC
TABLE 1: Boundary conditions.

\begin{tabular}{lcc}
\hline Component & Boundary conditions & Magnitude \\
\hline \multirow{2}{*}{$\begin{array}{l}\text { Mainstream } \\
\text { inlet }\end{array}$} & Mass flux rate & $32.72(\mathrm{~kg} / \mathrm{s})$ \\
& Gas temperature & $1300(\mathrm{~K})$ \\
& Turbulent intensity & $5(\%)$ \\
& Hydraulic diameter & $0.324(\mathrm{~m})$ \\
\hline \multirow{2}{*}{ Mainstream } & Pressure & $1.573(\mathrm{MPa})$ \\
& Turbulent intensity & $5(\%)$ \\
& Hydraulic diameter & $0.324(\mathrm{~m})$ \\
Coolant & Air temperature & $300(\mathrm{~K})$ \\
chamber & Pressure & $1.82138(\mathrm{MPa})$ \\
& Pressure recovery & 0.98 \\
& coefficient & $10(\%)$ \\
& Turbulent intensity & $0.01026(\mathrm{~m})$ \\
\hline \multirow{2}{*}{ Droplets } & Hydraulic diameter & $5(\mathrm{um})$ \\
& Diameter & $0.003(\mathrm{~kg} / \mathrm{s})$ \\
\hline
\end{tabular}

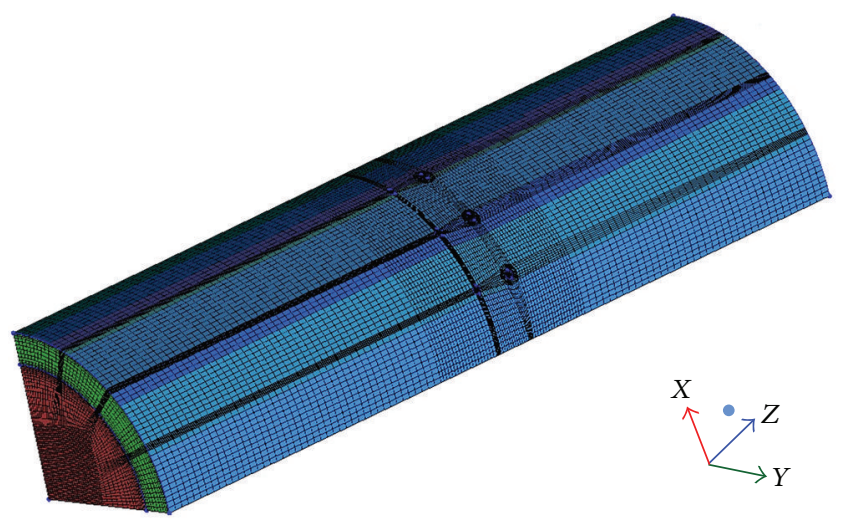

Figure 2: Meshes.

cluster with sixteen Pentium-4 3.0 GHz personal computers. The convergence criteria of the steady-state solution are judged by the reduction in the mass residual by a factor of 6 , typically, in 2000 iterations.

\section{Results and Discussion}

Based on the reliable computation model, the results obtained with different pin fin diameter ratios $D / d$ and different detached spaces from pin fin array to impinging holes array $L$ are presented in order to validate the CFD simulation mentioned above so that the performance of introducing pin fin to impinging structure would be studied well. In order to be convenient for analysis description, cases for different $L$ and $D / d$ are written as Case 1 to Case 10 shown in Table 2.

4.1. Flow Structure. Figure 3 shows a sequence of the streamwise velocity magnitude contours along the $z$-axis in various cases. The geometric center of the impinging jet in the coolant chamber is represented by the red region, which is coolant 


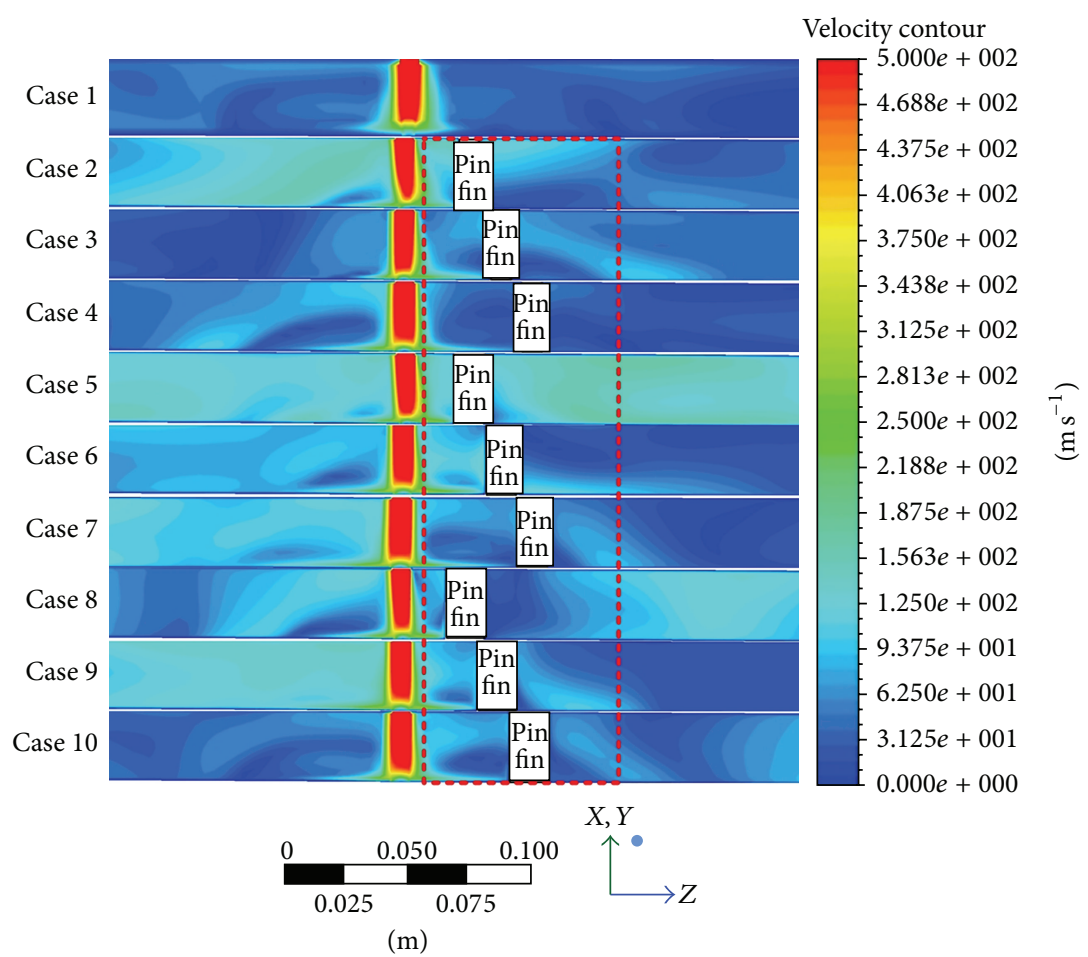

FIgURE 3: Contours of velocity.

TABLE 2: Cases number for different geometric parameters.

\begin{tabular}{lcc}
\hline Case & $D / d$ & $L(\mathrm{~mm})$ \\
\hline 1 & & no pin fins \\
2 & 1 & \\
3 & 1 & 34 \\
4 & 1 & 51 \\
5 & 1.5 & 68 \\
6 & 1.5 & 34 \\
7 & 1.5 & 51 \\
8 & 2 & 68 \\
9 & 2 & 34 \\
10 & 2 & 51 \\
\hline
\end{tabular}

holes at $Z=525 \mathrm{~mm}$. After the coolant strikes the inner wall, there are vortexes formed. The jet impingement and the vortex formed out of the coolant flow cool the surface of the inner wall. The impinging flow does not detach from the wall but creates three regions around the impinging jet, which are called free jet region, stagnation flow region, and wall jet region, just as is mentioned in [24]. The coolant-affected wall jet region is displayed as light-colored area, which can be recognized around the red free jet region from each of the Cases 1 to 10. And the cold film layer with a thickness of about half jet diameter blankets the inner wall surface quite efficiently around the wall jet region.

Case 1 was set to be a cooling structure without pin fins. Comparing Case 1 with other cases in Figure 3, it can be obviously found that most of those cases have more faster velocity areas in the coolant chamber than that in Case 1. With speed increasing, higher convective heat efficiency can be obtained due to more heat that had gone away with the coolant.

In the red dashed wireframe of Figure 3, local region comparison among Cases $2-4$, which have the same $D / d=$ 1 , presents the different flow structure around the pin fin under the circumstances of three sorts of $L$. Note that after the coolant strikes the inner wall, it meets the pin fin and is obstructed and separated by it; thus vortexes take shape between the outer wall and inner wall. So that coefficient of heat transfer were increased with the increasing of the tempestuously flow of the vortexes. But in contour of Case 4 , velocity in the upstream flow of the pin fins is not faster than that in the downstream. That is because when the pin fins are shifted downstream from the geometric center of the jet, the space between impinging hole and pin fin gets further so that the fast coolant could not get in touch with the pin fin. In a nutshell, the rising of distance $L$ clearly reduces the role of pin fins in forming turbulence for $D / d=2$. But, for Cases $8-10$ while $D / d=2$ as well as Cases $5-7$ when $D / d=1.5$, the median of $L$ could be the best because turbulence formed is more sufficient when $L=51$ seen in the contours.

4.2. Inner Wall Temperature and Cooling Effectiveness. Figure 4 shows a comparison of temperature distribution on the inner wall with a group of changing parameters, which is the conventional model in actual situations. This figure shows that the temperature at the stagnation flow region is much the same among the cases with pin fins, while the 


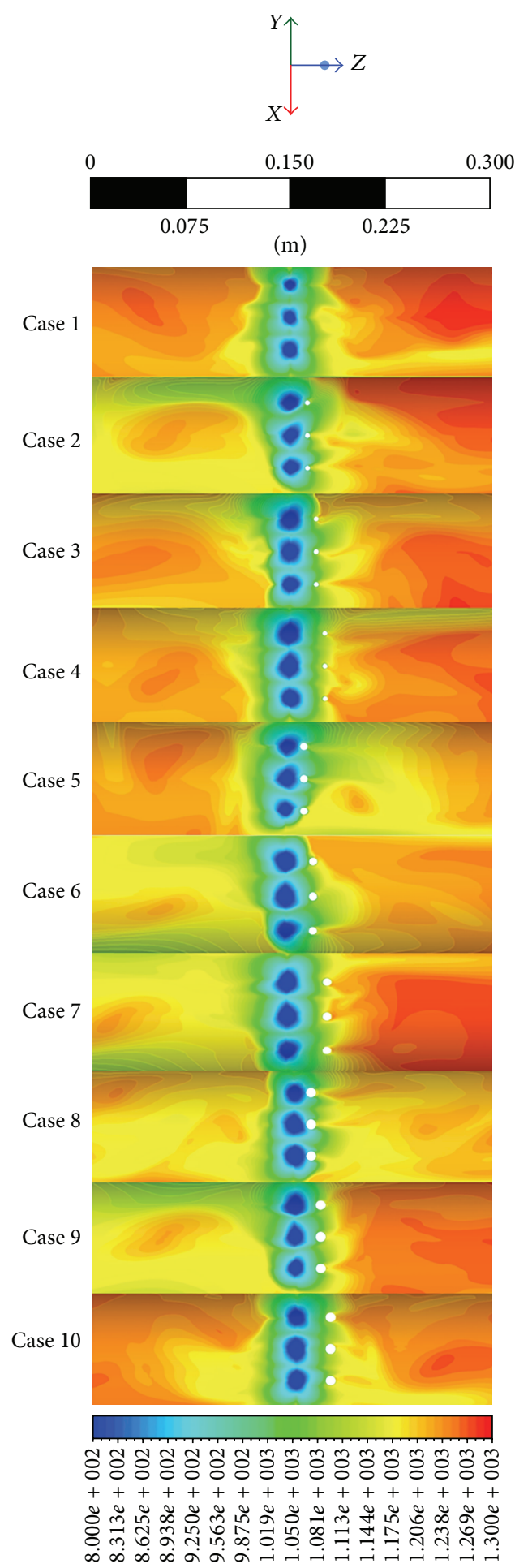

(K)

FIGURE 4: Contours of inner wall temperature.

lower temperature area (blue region) is larger than that in Case 1, which illustrates that the combination of impinging holes and pin fins has a good effect on reducing temperature in the stagnation flow region. For all the Cases from 1 to 10 , the different colors distribution represents corresponding temperature which is described in detail on the temperature legend. Overall, it could be concluded that the yellow area in Cases 2-10 is larger than that in Case 1; that means the pin fin structure imported to the impingement cooling style enhances the impinging cooling effectiveness. 


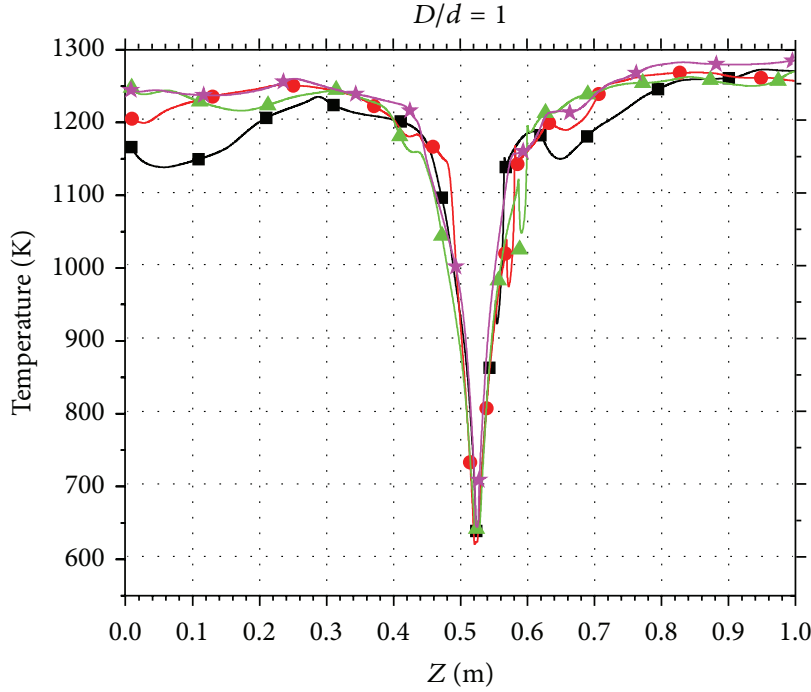

(a)

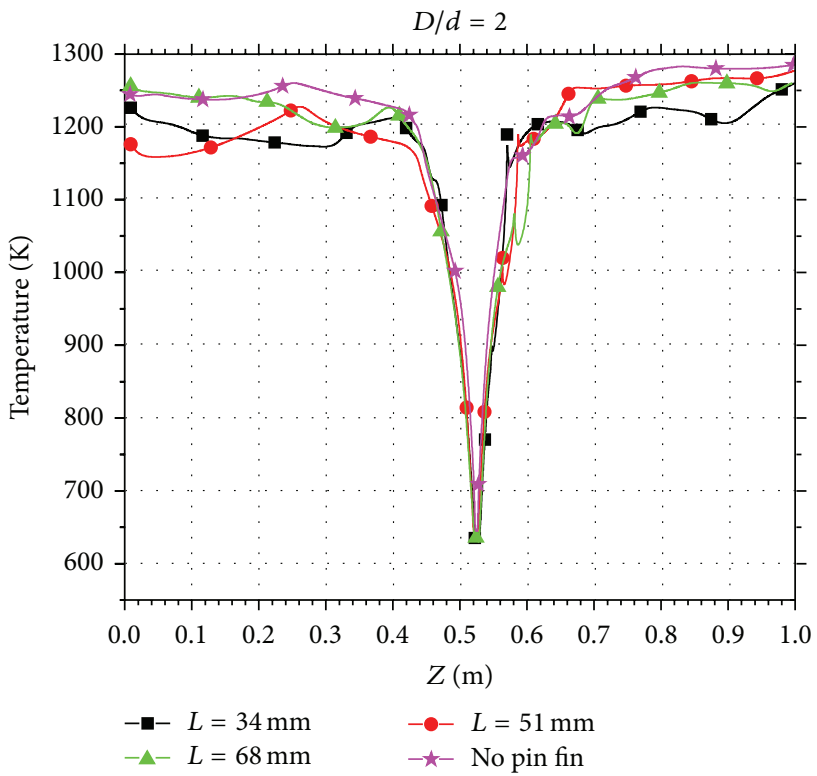

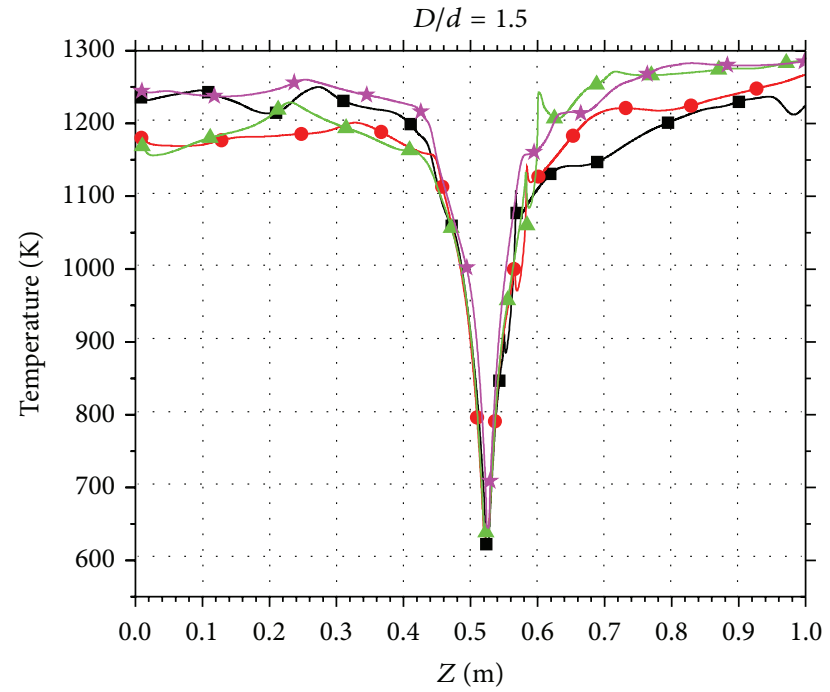

(b)

(c)

Figure 5: Comparison curves of $T_{w}$ in Line 1.

One important factor that must be considered in the design of pin fin is the detached spaces from pin fin array to impinging holes array. The pin fins should be placed in a suitable position in the downstream flow in order to acquire enough turbulence. Therefore, Figures 5(a), 5(b), and 5(c) provided temperature values along $Z$ direction at Line 1 , which is in the middle of the inner wall just as shown in Figure 5. They represent temperature comparison of three kinds of $L$ in different $D / d$ cases separately. It can be observed from Figure 5(a) that the temperature becomes lower with the decrease in detached space, and the lowest temperature is obtained when $L=34 \mathrm{~mm}$. The $L=34 \mathrm{~mm}$ cases also can be proved to be the best distance in Figure 5(c). But in Figure 5(b) there is a little variation in the upstream where $Z$ belongs to $0-0.5 \mathrm{~m}$, and the cases with distance of $L=51 \mathrm{~mm}$ could get a lower average temperature.

The cooling effectiveness $(\eta)$ [22] is used to examine the performance of impinging cooling. The definition of $\eta$ is

$$
\eta=\frac{T_{m}-T_{w}}{T_{m}-T_{c}},
$$

where $\eta$ is impinging cooling effectiveness, $T_{m}$ is mainstream temperature, $T_{w}$ is absolute temperature on the inner wall, and $T_{c}$ is the temperature of coolant. The definition provides an appropriate parameter for investigating impinging cooling when different pin fin structures are employed. Figure 6 shows the value profile of $\eta$, through the comparison of Cases 2, 3, and 4, and it is obvious that appropriate increase 


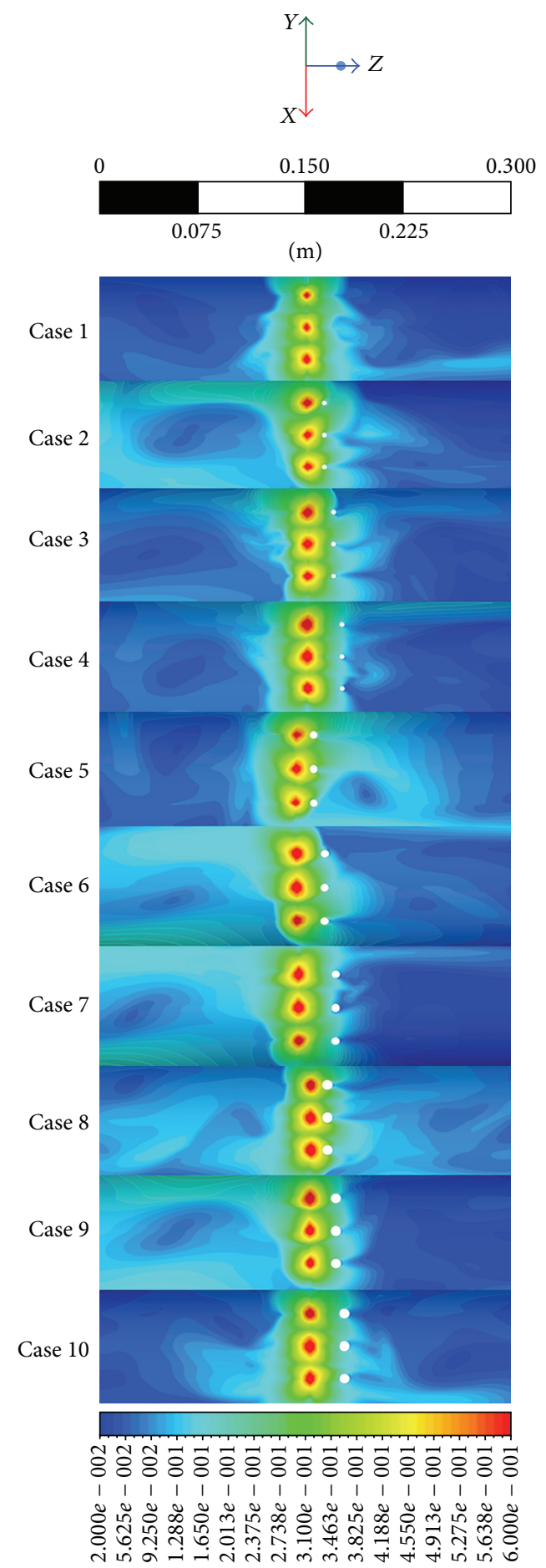

Figure 6: Contours of cooling effectiveness.

in the diameter of pin fins brings more area of higher $\eta$. Since the contact area between the coolant and the pin fin is increasing along with the $D / d$ augmenting, bring about more heat transmisson. But it cannot fairly show the cooling enhancement induced by pin fins because Case 6 seems to have the highest value of cooling effectiveness. In other words, for different cases, a weighted variable should be imported to evaluate the comprehensive temperature on the inner wall.

Consequently, $T_{\text {ave }}$ is defined as area weighted average temperature on the inner wall; its expression is represented as follows:

$$
\eta=\frac{T_{m}-T_{w}}{T_{m}-T_{c}},
$$


where $A_{w}$ is the area of the inner wall, $i$ is the number of wall mesh, and $n$ is the total number of mesh.

The area weighted average temperature is used to study the value of comprehensive temperature, and the value of $T_{\text {ave }}$ is able to evaluate the performance of impinging cooling. For all the ten cases without mist, the minimum value of Tave is $1174.46 \mathrm{~K}$ appearing in Case 6, which agrees with the description of cooling effectiveness.

4.3. Effect of Mist Added in Impinging Coolant. Mist, with uniform droplet size and mass of $5 \mu \mathrm{m}$ and $0.003 \mathrm{~kg} / \mathrm{s}$, is injected to the cooling chamber together with the air from the inlet surface. As documented and mentioned in the introduction, injecting mist into the air can improve the effect of cooling. The small water droplets' trajectories from the coolant inlet can be seen in Figure 7.

Figure 7 shows that the droplets impact the inner wall which moved with the coolant jet from the impinging hole. All the droplets evaporate in the domain between $Z=$ $350 \mathrm{~mm}$ and $Z=700 \mathrm{~mm}$ along the $z$-axis in the cooling chamber. However, the droplets exit in different times in different cases and have some transformation in different pin fin structures. Therefore, the distance moved by droplets could generate different performance of cooling and temperature distribution.

To explain the effect of mist added in the impinging cooling, comparison of the $T_{\text {ave }}$ in different cases is given in Figure 8 including all the cases with and without mist. Further data analysis in Figure 8 shows that after the mist is taken into impinging cooling chamber the value of Tave has a decreased range from $0.19 \mathrm{~K}$ to $26.86 \mathrm{~K}$ compared with the situation without mist. Through the combination of mist cooling and impinging cooling, the inner wall obtained $8.96 \mathrm{~K}$ of the average $T_{\text {ave }}$ decrease. It is observed that the best case without mist is the Case 6 indeed as mentioned in previous section, and the value is $1174.46 \mathrm{~K}$, which has a decrease of $38.77 \mathrm{~K}$ than Case 1 . As the same, when droplets are injected into the coolant, Case 6 still obtained the lowest area weighted average temperature.

A more data analysis reveals that there is no obvious pressure drop while importing various pin fin structures and taking mist into the coolant. In consequence it is a good method to get a lower temperature through using pin fin structure and mist cooling without excess pressure loss.

\section{Conclusion}

A one-fourth cylinder double chamber model with a single row of impinging holes in the outer wall was simplified from the gas turbine transition piece, and its operating conditions selected in this paper are featured by high temperature, high pressure, and high velocity of coolant. A complete three-dimensional numerical simulation is employed with different pin fin structures adopted in the impinging cooling. Mist cooling technology taken into the impinging cooling is tested and investigated. And it has been proven that the pin fins could enhance the cooling effectiveness without excess pressure loss. The temperature could obtain a lower value
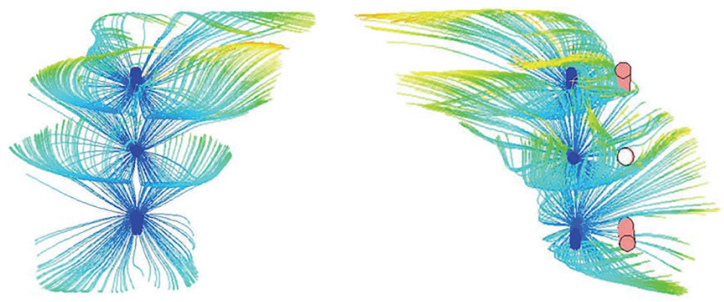

Case 1

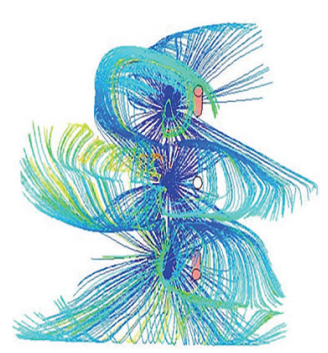

Case 6

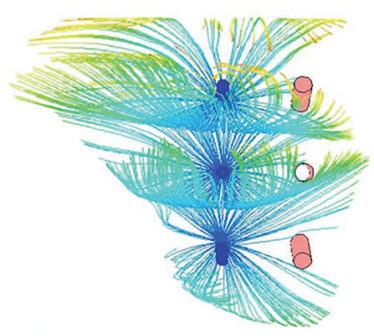

Case 2

Case 7

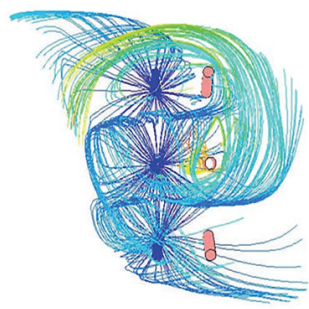

Case 3

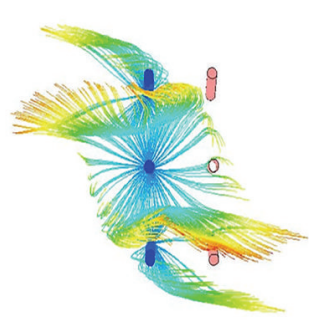

Case 4

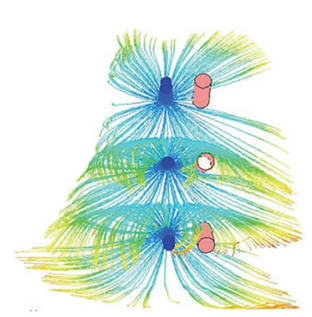

Case 5

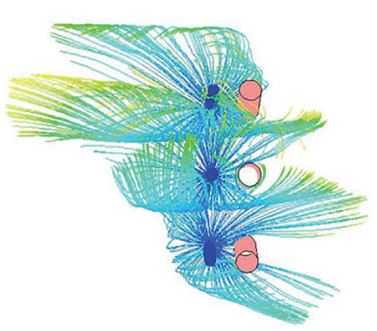

Case 8

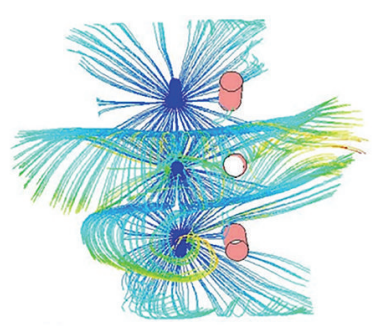

Case 9

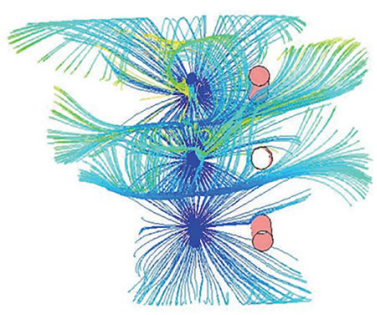

Case 10

FIGURE 7: Distributions of droplets particle track in different cases with uniform droplet mass and diameter.

when injecting mist into the cooling chamber. The optimal combination of the pin fin parameters and mist imported brings the optimum impinging cooling efficiency without excess pressure drop, and the lowest value of Tave has a decrease of $42.65 \mathrm{~K}$, which is a considerable value in cooling the hot components. That is to say the procedure and result of the CFD simulation in this paper have some practical value 


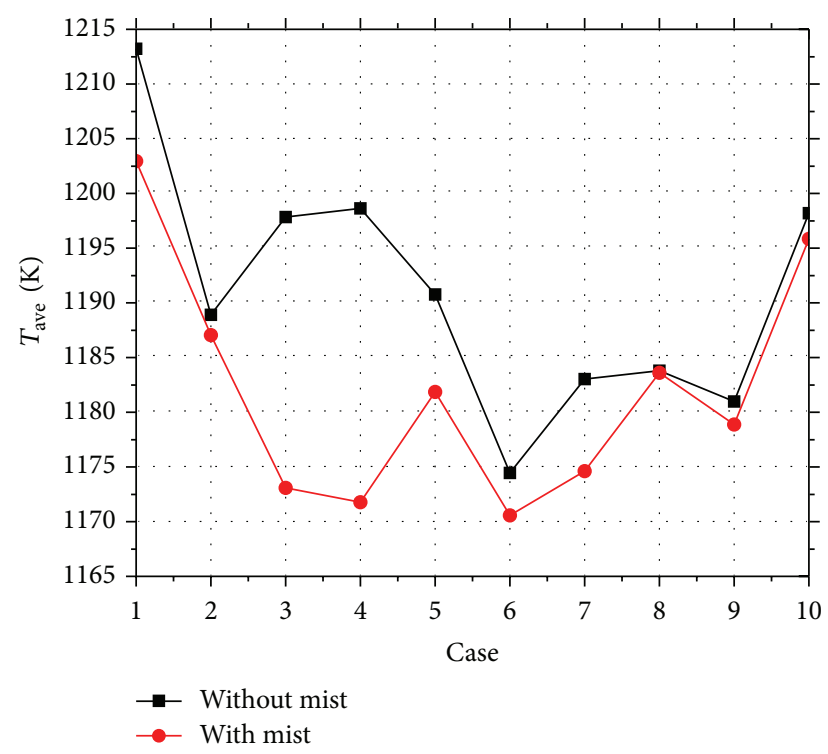

Figure 8: Comparison of the $T_{\text {ave }}$ value in different cases with or without mist.

for real processing and guiding significance for theory. The validation of the present study will be employed in further studies of impinging cooling with pin fins and mist cooling.

\section{Nomenclature}

$\begin{array}{ll}D: & \text { Diameter of pin fin } \\ d: & \text { Diameter of impinging hole } \\ L: & \begin{array}{l}\text { Detached spaces from pin fin array to } \\ \text { impinging holes array }\end{array} \\ A_{w}: & \text { Area of inner wall } \\ V_{i}: & \text { Volume of the no. } i \text { grid } \\ T_{w}: & \text { Absolute temperature on the inner wall } \\ T_{\text {ave }}: & \text { Area weighted average temperature on the } \\ P_{\mathrm{v}-\text { ave }}: & \text { inner wall of coolant chamber } \\ & \text { Volume weighted average pressure in the } \\ X, Y, & \text { coolant chamber }\end{array}$

$X, Y$, and $Z$ : Nondimensional coordinates directions.

\section{Greek Symbols}

$\eta$ : Cooling effectiveness.

\section{Suffixes}

$m$ : Mainstream flow

c: Coolant flow

aw: Adiabatic wall.

\section{Conflict of Interests}

The authors declare that there is no conflict of interests regarding the publication of this paper.

\section{Acknowledgments}

This research is supported by the Technology Development of Jilin Province (no. 20126001), the Plan for Scientific and Technology Development of Jilin Province (no. 20130522150JH), and the Fund for the Doctoral Program of Higher Education (no. 20130061120035).

\section{References}

[1] N. Zuckerman and N. Lior, "Impingement heat transfer: correlations and numerical modeling," Journal of Heat Transfer, vol. 127, no. 5, pp. 544-552, 2005.

[2] A. A. Tawfek, "Heat transfer studies of the oblique impingement of round jets upon a curved surface," Heat and Mass Transfer, vol. 38, no. 6, pp. 467-475, 2002.

[3] Z. L. Yu, T. Xu, J. L. Li, L. Ma, and T. S. Xu, "Comparison of a series of double chamber model with various hole angles for enhancing cooling effectiveness," International Communications in Heat and Mass Transfer, vol. 44, pp. 38-44, 2013.

[4] S. Göppert, T. Gürtler, H. Mocikat, and H. Herwig, "Heat transfer under a precessing jet: effects of unsteady jet impingement," International Journal of Heat and Mass Transfer, vol. 47, no. 1213, pp. 2795-2806, 2004.

[5] B. P. E. Dano, J. A. Liburdy, and K. Kanokjaruvijit, "Flow characteristics and heat transfer performances of a semi-confined impinging array of jets: effect of nozzle geometry," International Journal of Heat and Mass Transfer, vol. 48, no. 3-4, pp. 691-701, 2005.

[6] B. C. Y. Cheong, P. T. Ireland, J. P. C. W. Ling, and S. AshforthFrost, "Flow and heat transfer characteristics of an impinging jet in crossflow at low nozzle-to-plate spacings," in Proceedings of the ASME Turbo Expo, pp. 601-617, June 2005.

[7] J.-C. Han, "Turbine blade cooling studies at Texas A\&M University: 1980-2004," Journal of Thermophysics and Heat Transfer, vol. 20, no. 2, pp. 161-187, 2006.

[8] T. Horbach, A. Schulz, and H.-J. Bauer, "Trailing edge film cooling of gas turbine airfoils-external cooling performance of various internal pin fin configurations," Journal of Turbomachinery, vol. 133, no. 4, Article ID 041006, pp. 1-9, 2011.

[9] Y. Wang, F. Houshmand, D. Elcock, and Y. Peles, "Convective heat transfer and mixing enhancement in a micro-channel with a pillar," International Journal of Heat and Mass Transfer, vol. 62, pp. 553-561, 2013.

[10] Y. Rao, Y. Xu, and C. Wan, "An experimental and numerical study of flow and heat transfer in channels with pin fin-dimple and pin fin arrays," Experimental Thermal and Fluid Science, vol. 38, pp. 237-247, 2012.

[11] T. Wang and X. Li, "Mist film cooling simulation at gas turbine operating conditions," International Journal of Heat and Mass Transfer, vol. 51, no. 21-22, pp. 5305-5317, 2008.

[12] X. Li and T. Wang, "Simulation of film cooling enhancement with mist injection," Journal of Heat Transfer, vol. 128, no. 6, pp. 509-519, 2006.

[13] X. Li and T. Wang, "Effects of various modeling schemes on mist film cooling simulation," Journal of Heat Transfer, vol. 129, no. 4, pp. 472-482, 2007.

[14] Z. L. Yu, T. Xu, J. L. Li, T. S. Xu, and Y. Tatsuo, "Computational analysis of droplet mass and size effect on Mist/air impingement cooling performance," Advances in Mechanical Engineering, vol. 2013, Article ID 181856, 8 pages, 2013. 
[15] J. Benoit, C. Johnston, and M. Zingg, "Enhancing gas turbine power plant profitability: chronic transition piece and turbine part failures in some 501F gas turbines led to a replacement part redesign," Power Engineering, vol. 111, no. 11, pp. 140-144, 2007.

[16] A. Gallegos Muñoz, V. Ayala-Ramírez, J. A. Alfaro-Ayala, and B. M. Toledo Acosta, "Optimization of the transition piece applying genetic algorithms," Applied Thermal Engineering, vol. 31, no. 16, pp. 3214-3225, 2011.

[17] “ANSYS FLUENT 12.1 Documentation,” ANSYS, 2009.

[18] Z. Yu, T. Xu, J. Li, H. Xiu, and Yun Li, "Numerical Simulation on the Effect of Turbulence Models on Impingement Cooling of Double Chamber Model," Mathematical Problems in Engineering, vol. 2013, Article ID 170317, 8 pages, 2013.

[19] W. E. Ranz and W. R. Marshall Jr., "Evaporation from drops, part I," Chemical Engineering Progress, vol. 48, pp. 141-146, 1952.

[20] K. Y. Kuo, Principles of Combustion, John Wiley and Sons, New York, NY, USA, 1986.

[21] X. Li and T. Wang, "Effects of various modeling schemes on mist film cooling simulation," Journal of Heat Transfer, vol. 129, no. 4, pp. 472-482, 2007.

[22] G. Subbuswamy and X. Li, "Numerical study of film cooling enhancement in gas turbine combustor liner," in Proceedings of the ASME International Mechanical Engineering Congress and Exposition (IMECE '08), pp. 1237-1247, November 2008.

[23] http://www.matweb.com .

[24] B. Han and R. J. Goldstein, "Jet-impingement heat transfer in gas turbine systems," Annals of the New York Academy of Sciences, vol. 934, pp. 147-161, 2001. 


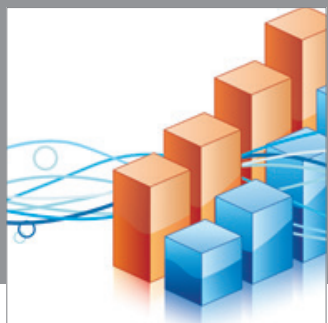

Advances in

Operations Research

mansans

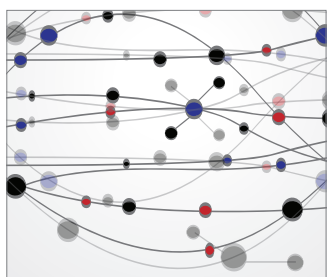

The Scientific World Journal
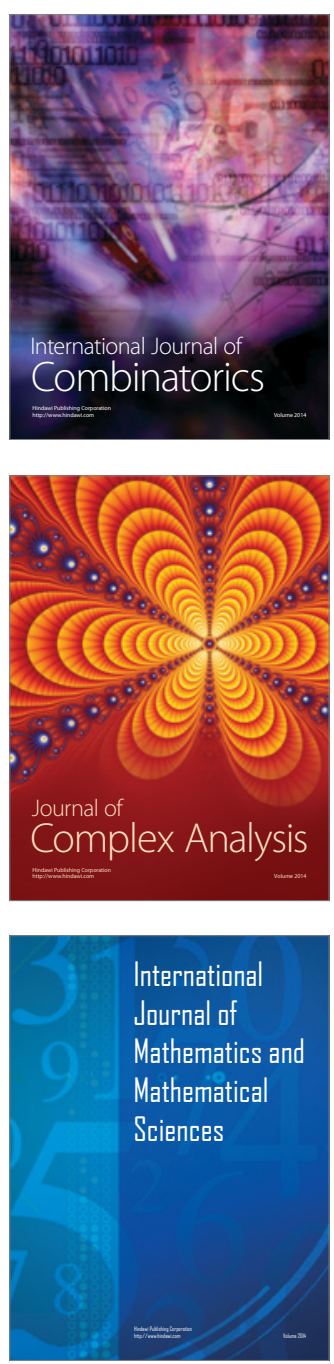
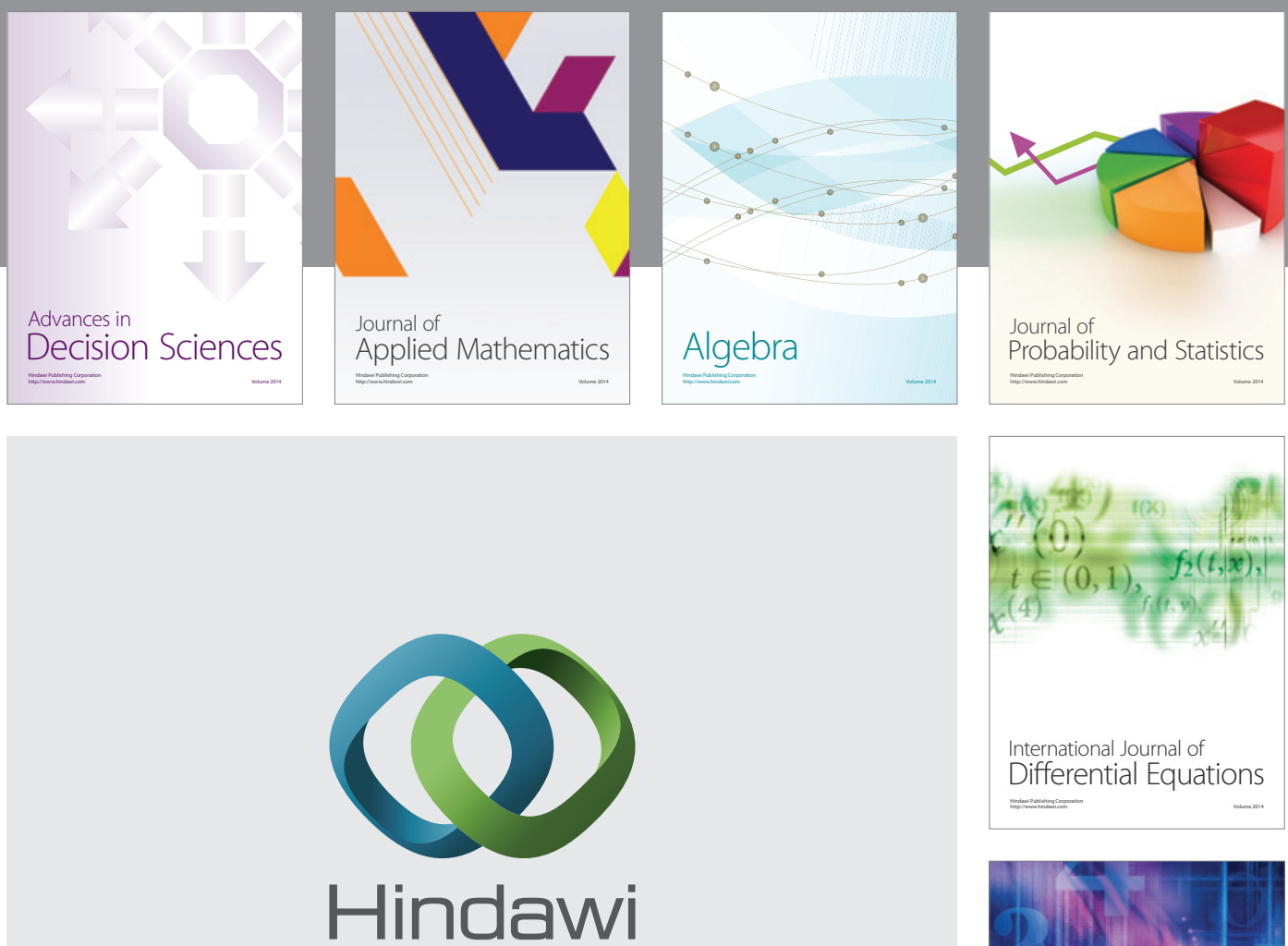

Submit your manuscripts at http://www.hindawi.com
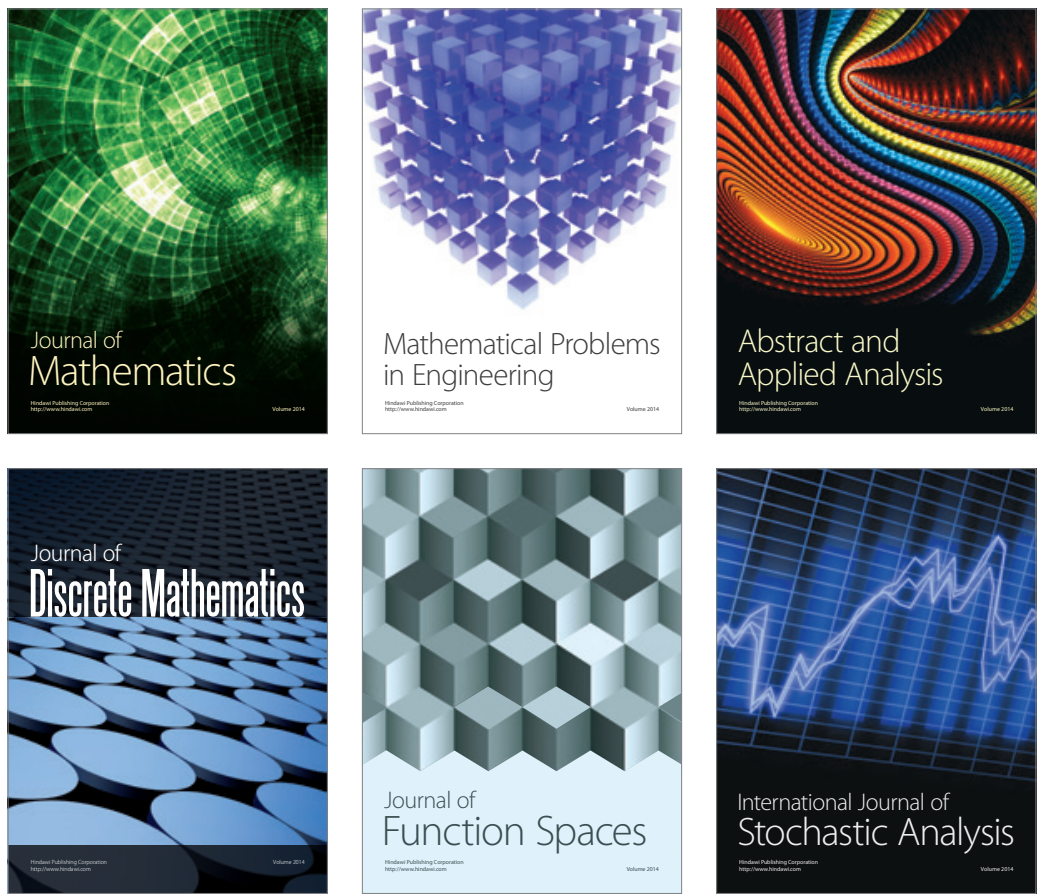

Journal of

Function Spaces

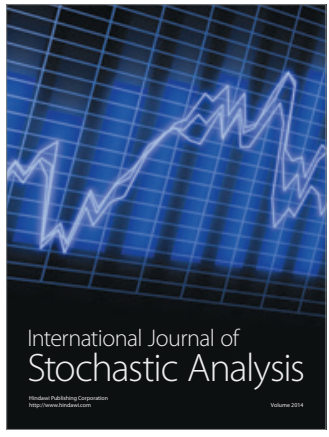

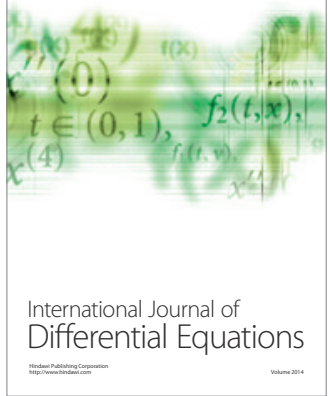
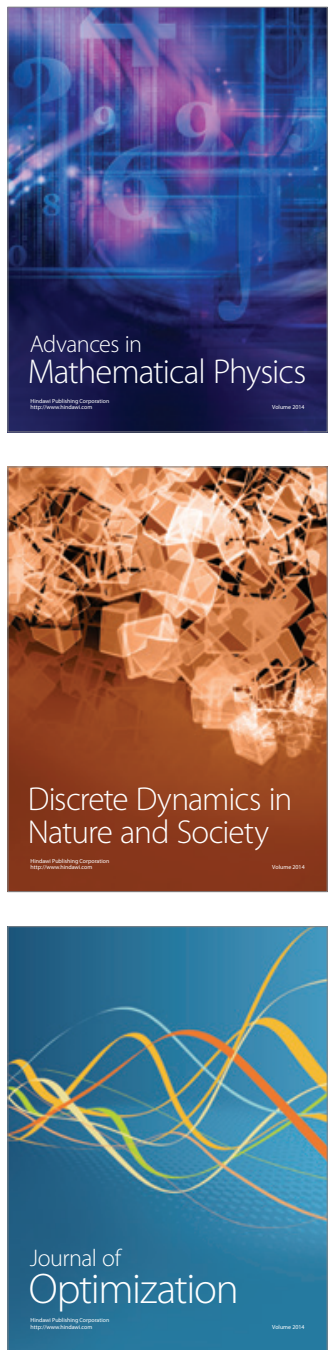